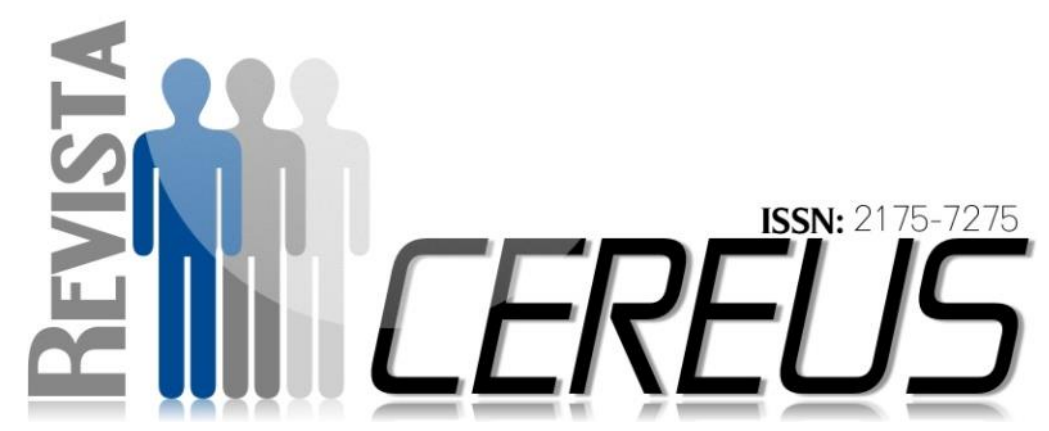

DOI:10.18605/2175-7275/cereus.v9n1p.106-117

\title{
POLUIÇÃO SONORA EM SETORES ESPECÍFICOS DO HOSPITAL REGIONAL DE GURUPI/TO: AVALIAÇÃO DOS PARÂMENTROS RECOMENDADOS PELAS NORMAS TÉCNICAS DE ACÚSTICA DA ABNT
}

FERNANDES, Anne Carolynne Fonseca ${ }^{1}$ TELES, Marcela de Barros ${ }^{2}$ BRITO, Marcineide Maria Veli da Silva ${ }^{3}$ MELO, Marllos Peres de ${ }^{4}$ AZEVEDO JUNIOR, Jairo ${ }^{5}$ SAKAY, Lady 6

\section{RESUMO}

O ambiente hospitalar é um local que necessita ser silencioso e tranquilo devido aos enfermos que ali se encontram. A circulação de pessoas da equipe multidisciplinar e o número considerável de equipamentos e alarmes sonoros deixam o ambiente ruidoso. O objetivo desta pesquisa foi mensurar os níveis de ruídos nos setores Clínica Cirúrgica, Clínica Médica, Pronto Socorro Adulto e Unidade de Terapia Intensiva do Hospital Regional de Gurupi/TO estão dentro dos valores

${ }^{1}$ Graduanda em Enfermagem no do Centro Universitário Unirg- fernandescarol@live.com ${ }^{2}$ Graduanda em Enfermagem no do Centro Universitário Unirg.-marcelinhaa.teles@gmail.com ${ }^{3}$ Especialista em Urgência e Emergência-marcineidebrito39@hotmail.com.

${ }^{4}$ Professor de bioestatística do Centro Universitário Unirg, Doutor em Agronomia pela UFTTO -marllosperes@gmail.com

${ }^{5}$ Doutor em produção animal pela Universidade Federal de Lavras. Professor de biologia celular do Centro Universitário Unirg

6 Doutora em educação pela UNB. Professora do Centro Universitário Unirg. 
recomendados pelas Normas Técnicas da Acústica da ABNT. Foi realizada uma pesquisa, descritiva e quantitativa através da análise e coleta de dados durante uma semana, durante 06 (seis) dias, sendo 02 (dois) dias em cada setor em diferentes horários, foi utilizado um decibelímetro para realizar mensurações a cada cinco segundos. Após a análise dos dados, verificou-se o valor máximo de $88 \pm 3,06 \mathrm{~dB}$ e o valor mínimo de 52,8 \pm 4,12 dB e que as médias gerais das avaliações foram na CC de $65,5 \mathrm{~dB}$; na CM de $65,3 \mathrm{~dB}$; no PSA de 70,1 dB; e na UTI de 65,5 dB. Pode-se observar que estes resultados estão acima do recomendado pela norma da acústica da ABNT.

Palavras-chave: Ambiente hospitalar; Acústica; Ruídos.

\section{SOUND POLLUTION IN SPECIFIC SECTORS OF} REGIONAL HOSPITAL OF GURUPI / TO: EVALUATION OF PARAMETERS RECOMMENDED BY ABNT'S TECHNICAL STANDARDS OF ACOUSTICS

\section{ABSTRACT}

The hospital environment is a place that needs to be quiet and peaceful due to the sick people who are there. The movement of people from the multidisciplinary team and the considerable number of sound equipment and alarms leave the environment noisy. The objective of this research was to measure if the noise levels in the Surgical Clinic, Medical Clinic, Adult Emergency Room and Intensive Care Unit of the Regional Hospital of Gurupi / TO are within the values recommended by the ABNT Acoustic Technical Standards. A descriptive and quantitative research was carried out through the analysis and data collection during a week, during 06 (six) days, being two (2) days in each sector at different times, a decibel meter was used to perform measurements every five seconds. After analyzing the data, the maximum value was $88 \pm 3.06 \mathrm{~dB}$ and the minimum value was $52.8 \pm 4.12 \mathrm{~dB}$ and that the general 
means of the evaluations were in the $\mathrm{CC}$ of $65.5 \mathrm{~dB}$; In $\mathrm{CM}$ of $65.3 \mathrm{~dB}$; In PSA of $70.1 \mathrm{~dB}$; And in the ICU of $65.5 \mathrm{~dB}$. It can be observed that these results are above that recommended by the acoustic standard of ABNT.

Keywords: Hospital environment; Acoustics; Noise. 


\section{INTRODUÇÃO}

A exposição excessiva a fontes de ruído pode gerar consequências psicológicas e físicas nem sempre reversíveis, incluindo perdas auditivas permanentes (SILVA et al., 2014).

A surdez permanente resulta de exposições prolongadas a níveis superiores a $80 \mathrm{~dB}$, bem como a sons de curta duração a mais de $110 \mathrm{~dB}$, ou ainda por acúmulo de fadiga auditiva sem tempo adequado de recuperação (MINISTÉRIO DA SAÚDE, 2015).

Um local que deveria ser adequado para uma pessoa que necessita de cuidados nem sempre está favorável, devido às situações que produzem frequências sonoras, trazendo irritabilidade e desconforto aos indivíduos que ali se encontram (OTENIO; CREMER; CLARO, 2007).

Embora o sono possa parecer minucioso, pode ter uma diferença tamanha em sua estabilidade. Por este motivo, refere-se à importância de um ambiente silencioso e tranquilo para benefício tanto da pessoa que está recebendo cuidados, quanto da equipe hospitalar, que são afetados com o cansaço, estresse e danos psicológicos e fisiológicos
(CARVALHO; PEDREIRA; AGUIAR, 2005).

Esta pesquisa visa a mudança nos hábitos que produzam ruídos em setores do hospital, resultando em maior ou menor bem-estar tanto dos pacientes quanto da equipe multidisciplinar a prevenção dessa exposição aos ruídos para que os mesmos sintam-se melhores para uma boa recuperação e jornada de trabalho.

O presente estudo teve por objetivo avaliar os níveis de pressão sonora encontrados em uma unidade hospitalar, em horários e locais diferentes, para verificação do cumprimento (ou não) da ABNT (NBR 10152/1987), que estabelece os níveis aceitáveis de ruído em diversos ambientes, inclusive no ambiente hospitalar.

No Brasil, para garantir o conforto acústico, a NBR 10152/1987 (Níveis de Ruído para Conforto Acústico, Associação Brasileira de Normas Técnicas, 1987) fixa os limites de ruído para diversos ambientes. A NBR 10152/1987 indica os níveis de pressão sonora permitida e as Curvas NC (noise criterion curves). 
Um ambiente pode ser classificado acusticamente por um número único obtido através das chamadas Curvas NC. Tais curvas foram desenvolvidas para análise de níveis de ruído de fundo (ROSA, 2003), sendo que uma dada NC pode corresponder a diferentes níveis de pressão sonora, conforme a frequência do som.
Conforme a NBR 10152/1987, em ambientes hospitalares, os níveis de pressão sonora devem variar entre 35 e $45 \mathrm{~dB}$, encontrando-se entre as curvas NC30 e NC50, conforme apresentado na Tabela $1 . \quad \mathrm{O}$ valor inferior representa o nível sonoro para conforto, e o valor superior corresponde ao nível sonoro máximo considerado aceitável para o ambiente.

Tabela 1 - Níveis sonoros recomendados conforme o ambiente $(\mathrm{dB}(\mathrm{A})$ e NC)

\begin{tabular}{lll|ll}
\hline \multicolumn{1}{c|}{ Ambiente hospitalar } & $\mathrm{dB}(\mathrm{a})$ & \multicolumn{2}{c}{$\mathrm{NC}$} \\
\hline Apartamentos, Enfermarias, Berçários, Centros cirúrgico & 35 & 45 & 30 & 40 \\
Laboratórios, Área de uso público & 40 & 50 & 35 & 45 \\
Serviços & 45 & 50 & 40 & 50 \\
\hline
\end{tabular}

Fonte: ABNT - NRB 10152/1987

O ambiente hospitalar é um espaço onde o objetivo é cuidar do paciente, buscando sempre sua melhora de todas as formas possíveis, e para isso, contam com os diversos aparelhos modernos ou não, que são capazes de auxiliá-los nessa busca.

Neste local, há pessoas que necessitam de atendimento especializado com vigilância por 24 horas e outras que necessitam apenas de cuidados básicos. Porém, os dois casos utilizam a assistência multidisciplinar, interdisciplinar e os recursos especializados (DIAS, MATTA, NUNES, 2006).
Alguns fatores são estressantes durante uma internação hospitalar. Os hospitais deveriam ser locais silenciosos e tranquilos para que assim se promova a saúde com mais rapidez e eficácia, porém sabe-se que esta não é a realidade atualmente, o que atrapalha no conforto dos enfermos que respondem com medo, ansiedade, distúrbios do sono, comprometimento cognitivo e dor (NETO et al., 2010).

De acordo com Morton e Fontaine (2011), "O estresse é definido com uma situação em que o organismo se vê diante de um 
estímulo que causa desequilíbrio entre - funcionamento psicológico e fisiológico."

A mobilização da equipe que necessita de agilidade para uma eventual intercorrência, contribui com os ruídos, além dos aparelhos que são utilizados durante todo o processo para a estabilização do paciente, como por exemplo, aspiração de vias aéreas superiores (SANTOS, 2010).

O convívio dentro deste local pode parecer mecânico, ou totalmente técnico, sem levar em consideração qualquer tipo de emoção, e o método baseado na realização de tarefas pode resultar em um afastamento entre equipe, paciente e familiar, sendo utilizado como artifício, devido ao cansaço e sobrecarga de trabalho (WEICH et al., 2011).

Devido alguns não ter bom senso levam para 0 ambiente de trabalho seus aparelhos celulares e

\section{MATERIAIS E MÉTODOS}

Foi realizada uma pesquisa, descritiva e quantitativa através da análise e coleta de dados do Hospital Regional de Gurupi/TO, localizado na Rua Presidente Juscelino Kubitscheck, n 1541 - setor Central, durante 06 utilizam o mesmo gerando algum escape de som. Outros fatores como: troca de turnos, limpezas, movimento de equipamentos, a água caindo da pia e o simples abrir e fechar da porta (MANZINI, 2015).

Todos estes fatores influenciam na recuperação, pois o cotidiano do indivíduo é interrompido devido à doença, como o sono que é de extrema importância. Cada pessoa necessita ser vista de forma individualizada, pois ninguém tem o mesmo padrão de sono. $\mathrm{E}$ a enfermagem deve avaliar cada um para ajudá-lo a manter seu ciclo de sono-vigília.

A qualidade no sono é importante para as funções fisiológicas, comportamentais e biológicas, e pacientes que não a mantêm recuperam-se de forma lenta (POTTER, PERRY, 2013).

(seis) dias, sendo 02 (dois) dias em cada setor em diferentes horários, onde foi verificado o nível de ruído de acordo com (TABELA 2):

Local : 
CME: Clínica Médica Horários Extras Equipe/Paciente

CMPPM: Clínica Médica Passagem de Plantão Matutino

CMPPN: Clínica Médica Passagem de Plantão Noturno

CMV: Clínica Médica Horário de Visita CCE: Clínica Cirúrgica Horários Extras Equipe/Paciente

CCPPM: Clínica Cirúrgica Passagem de Plantão Matutino

CCPPN: Clínica Cirúrgica Passagem de Plantão Noturno

CCV: Clínica Cirúrgica Horário de Visita

PSAE: Pronto Socorro Adulto Horários Extras Equipe/Paciente
PSAPPM: Pronto Socorro Adulto Passagem de Plantão Matutino PSAPPN: Pronto Socorro Adulto Passagem de Plantão Noturno PSAV: Pronto Socorro Adulto Horário de Visita

UTIE: Unidade de Terapia Intensiva Horários Extras Equipe/Paciente

UTIPPM: Unidade de Terapia Intensiva Passagem de Plantão Matutino

UTIPPN: Unidade de Terapia Intensiva Passagem de Plantão Noturno

UTIV: Unidade de Terapia Intensiva Horário de Visita

Tabela 2 : Horários de mensuração dos ruídos

\begin{tabular}{|c|c|c|c|c|}
\hline SETOR & $\begin{array}{c}\text { PASSAGEM DE } \\
\text { PLANTAO } \\
\text { MATUTINA }\end{array}$ & $\begin{array}{c}\text { HORÁRIO DE } \\
\text { VISITAS }\end{array}$ & $\begin{array}{c}\text { PASSAGEM DE } \\
\text { PLANTÂO } \\
\text { NOTURNA }\end{array}$ & $\begin{array}{c}\text { HORÁRIO EXTRA } \\
\text { EQUIPE/PACIENTE }\end{array}$ \\
\hline CM & $7: 00$ as $8: 00$ & $15: 00$ as $16: 00$ & $19: 00$ as $20: 00$ & $8 \mathrm{~h}$ \\
\hline CC & $7: 00$ as $8: 00$ & $15: 00$ as $16: 00$ & $19: 00$ as $20: 00$ & $20 \mathrm{~h}$ \\
\hline PSA & $7: 00$ as $8: 00$ & $15: 00$ as $16: 00$ & $19: 00$ as $20: 00$ & $18 \mathrm{~h}$ \\
\hline UTI & $7: 00$ as $8: 00$ & $15: 00$ as $16: 00$ & $19: 00$ as $20: 00$ & $16 \mathrm{~h}$ \\
\hline
\end{tabular}

O instrumento da medição do ruído foi o Medidor de Nível de Pressão Sonora (MNPS) conhecido como decibelímetro, ITDEC 4000 da marca INSTRUTEMP, que foi fornecido às pesquisadoras pelo Departamento de Posturas e Edificações da Prefeitura de Gurupi.
O aparelho foi posicionado de acordo com a NBR 10152 - ABNT (1990), a uma distância de 1m de superfícies, com taxa de medição de 30 a $130 \mathrm{~dB}(\mathrm{~A})$.

Este hospital foi selecionado por ser referência de 17 cidades da região 


\section{RESULTADOS E DISCUSSÃO}

Foi verificado na pesquisa no Hospital Regional de Gurupi (FIGURA 1), que os níveis de pressão sonora observados variaram entre $63,7 \mathrm{~dB}$ na clínica médica em horário extra (CME) a $71,6 \mathrm{~dB}$ no posto de saúde durante a passagem de plantão.

Sendo que os menores valores médios de ruídos de acordo com os locais foram encontrados na CCPPN (64,8 dB), CME (63,7dB), PSAE $(69,0$ dB) e UTIE $(64,2 \mathrm{~dB})$.

Figura 1: Níveis de mensuração de acordo com o local e horário no hospital regional de Gurupi.

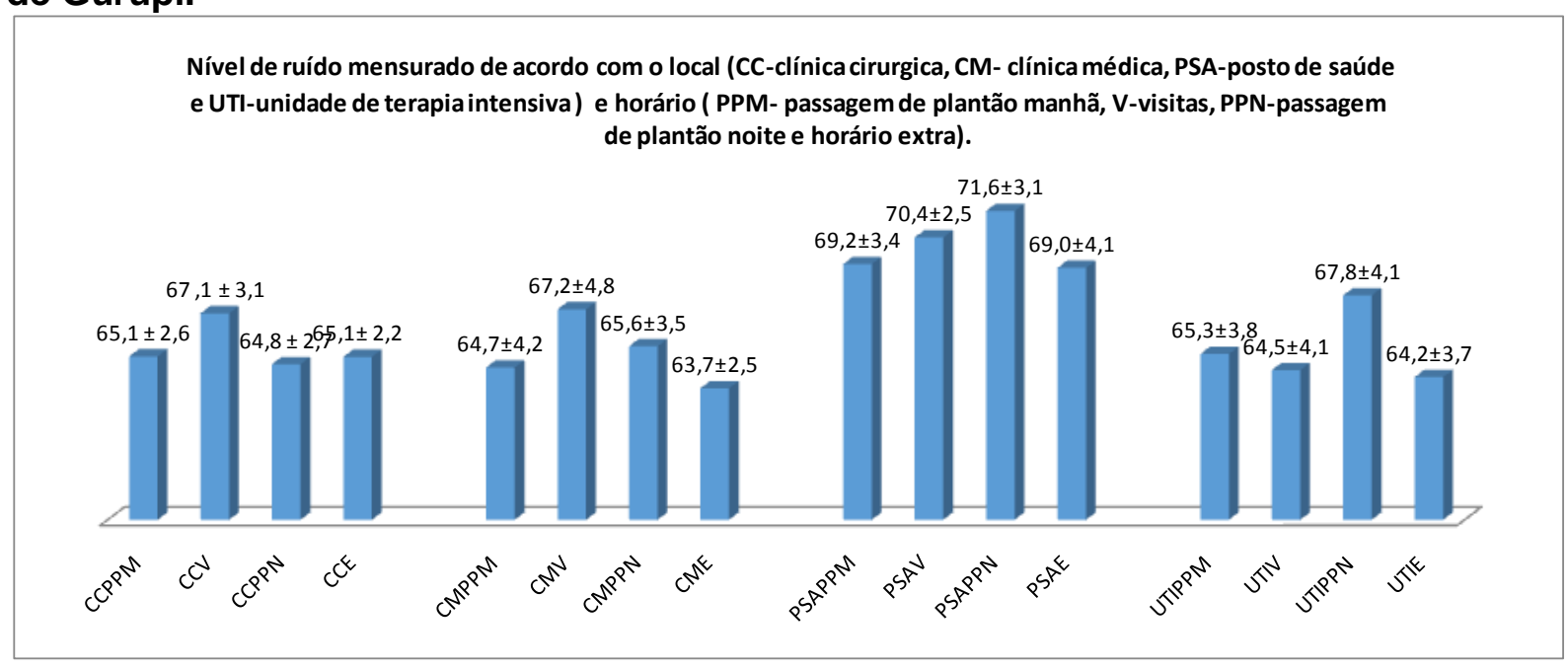

A NR 32 (2002) que dispõe sobre Saúde no Trabalho em Estabelecimentos de Assistência à Saúde informa que "devem ser atendidas condições de conforto relativas aos níveis de ruído de acordo com os estabelecido na NBR 10152, norma brasileira registrada no INMETRO". De acordo com a NBR 10152 - ABNT, o recomendado para ambiente hospitalar varia de 35 a 45 $\mathrm{dB}$.

Para os diferentes ambientes hospitalares, a ABNT recomenda 35 $\mathrm{dB}$ no período noturno e $45 \mathrm{~dB}$ no período diurno como níveis aceitáveis de ruídos. Neste trabalho, todas as aferições realizadas encontraram-se acima dos valores permitidos.

A partir de $55 \mathrm{~dB}$ a OMS recomenda algum tipo de proteção Rev. Cereus, v. 9, n. 1, p.106-117 , jan-abr./2017, UnirG, Gurupi, TO, Brasil. 
acústica para profissionais. No entanto, a norma regulamentadora do Ministério da Saúde NR17(Brasil 1999) preconiza que para 0 trabalho ser seguramente executado, o ruído aceitável deve ser de no máximo 65 $\mathrm{dB}$. No presente estudo, valores acima deste foram encontrados.

A média de ruído verificada durante todo este estudo foi de $66,6 \pm$ 3,4 dB. Valores semelhantes foram encontrados em hospitais na Áustria (60 a $65 \mathrm{~dB}$ ), Espanha ( $55 \mathrm{~dB})$, Itália (56,9 a 61,2 dB) e na Grécia (60,3 a $67,4 \mathrm{~dB}$ ), evidenciando que a poluição sonora não é apenas um problema local, envolvendo diversos países e culturas.

Pereira et al 2003, encontraram uma média geral de ruído na UTI de $65,36 \mathrm{~dB}$, verificando que o período noturno apresentou ruídos menos intensos que o período diurno, no trabalho em questão, apenas na Clínica cirúrgica (CCPPN), os resultados encontrados foram semelhantes aos encontrados por Pereira et al 2003, já que nos demais locais os ruídos mais intensos durante a noite.

Torres et al., 2007 observaram valores para os períodos matutino e noturno em UTIs equivalentes a 72,25
$\mathrm{dB}$ e $65 \mathrm{~dB}$, respectivamente. Otenio et al.,2007 utilizando doze medidas a cada hora, no período de 7 às 19 horas, com tempo médio de um minuto, obtiveram uma média de ruído de $62,7 \mathrm{~dB}$, variando entre 58 e $65 \mathrm{~dB}$.

No presente estudo, a UTI foi encontrada uma média de ruído no período da manhã de $66,3 \mathrm{~dB}$ e de $67,8 \mathrm{~dB}$ durante a noite, fato que pode ser justificado pelo maior número de profissionais no salão da UTI, em virtude da passagem de plantão.

Apesar da diferença encontrada nas passagens de plantão noturna e diurna $(67,8$ vs $65,3 \mathrm{~dB}$, respectivamente) não representar uma variação que possa ser considerada como clinicamente relevante, a disparidade verificada em relação aos valores recomendados justifica uma preocupação com a prevenção destes ruídos.

Acredita-se na hipótese de que o aumento no número de profissionais nestes momentos pode ter contribuído para gênese destes ruídos e que muitos dos pacientes ainda estão despertando pela manhã e a rotina normal na UTI ainda não tenha começado, enquanto á noite, esta passagem de plantão ocorre pouco tempo antes do horário de visita 
noturna, onde os pacientes muitas vezes estão recebendo os últimos cuidados relacionados à higiene pessoal, a fim de receber seus familiares.

As médias gerais das avaliações foram: na CC de 65,5 dB; na CM de 65,3 dB; no PSA de 70,1 dB; e na UTI de $65,5 \mathrm{~dB}$. Pode-se observar que estes resultados estão acima do recomendado pela norma da acústica da ABNT. Conforme Muniz (2009) em sua pesquisa, a média foi de $71 \mathrm{~dB}$, mínima de 55,6 dB e máxima de 89,3 $\mathrm{dB}$.

Ruído excessivo também foi observado em um estudo realizado no Hospital de São Paulo onde o nível de pressão sonora encontrado apresentou média de 65,3 dB, variando de 62,9 dB a 69,3 dB (PEREIRA et al, 2003).

Em Jundiaí - São Paulo os valores encontrados durante as aferições dos níveis de pressão sonora foram de $64,1 \mathrm{~dB}$ na primeira UTI, 58,9 dB na UCO (Unidade Coronariana) e $64 \mathrm{~dB}$ na segunda UTI (MARCEDO et al, 2009).

Notou-se que a maioria dos ruídos em todos os locais aumentava na hora da aspiração de pacientes, o aumento é inevitável na hora dos procedimentos. A parte de serviços do hospital também contribuía bastante, devido aos equipamentos utilizados. Analisando as vozes dos profissionais no ambiente, a voz masculina devido ao grave subia mais os decibéis, do que o das vozes femininas.

Portanto, através de estratégias simples e divulgação de informações, é possível proporcionar um ambiente mais tranqüilo e propício à recuperação dos pacientes das unidades de cuidados intensivos, bem como também proporcionar um ambiente de trabalho de melhor qualidade a todos os profissionais que nelas atuam.

Estes altos valores interferem na recuperação dos pacientes que estão debilitados, pois necessitam de um tratamento com qualidade $\mathrm{e}$ tranquilidade. O sono é de extrema importância para os mesmos, assegurando uma melhora mais rápida, diminuindo seu tempo de internação.

Portanto, cabe ao hospital tomar as devidas providencias, organizando palestras e orientações para toda sua equipe, pacientes e familiares para conscientização da importância do silêncio. E mais, fornecendo protetores de ouvidos aos profissionais para 


\section{CONCLUSÃo}

Para o hospital analisado, o nível de ruído verificado em todos os setores pesquisados estava consideravelmente acima do recomendado pelas normas da ABNT.

\section{REFERÊNCIAS}

MORTON, Patrícia Gonce; FONTAINE, DorrieK.Cuidados críticos de enfermagem: uma abordagem holística. Rio de Janeiro: Guanabara Koogan, 2011.

MUNIZ, Lediane Matos Nunes; STROPPA, Maria Aparecida.Desconfortos dos pacientes internados na UTI, quanto a poluição sonora. [online] Minas Gerais, 2009

NETO, Rui de Alencar Sampaio; MESQUITA, Fabrício Olinda de Souza; JUNIOR, Marçal duval Siqueira Paiva; RAMOS, Francimar Ferrari; ANDRADE, Flávio Maciel Dias de; JUNIOR, Marco Aurélio de ValoisCorreia.Ruídos na unidade de terapia intensiva: quantificação e percepção dos profissionais de saúde. Rev. bras. ter. intensiva [online]. 2010, vol.22, n.4, pp.369-374.

Norma Regulamentadora N. 32 - Segurança e Saúde no Trabalho em Estabelecimentos de Assistência à Saúde. [online] PORTARIA N. ${ }^{\circ} 37, \mathrm{DE} 06 \mathrm{DE}$ DEZEMBRO DE 2002.http://www.areaseg.com/nrindex/nr32.html.

OTENIO MH, CREMER E, CLARO EMT. Intensidade de ruído em hospital de 222 leitos na 18 ${ }^{\text {a }}$ Regional de Saúde - PR. RevBrasOtorrinolaringol. 2007;73(2):24550.

PADILHA, Kátia Grillo et al. Enfermagem em UTI: Cuidando do paciente crítico. Série enfermagem. Manole, 2009

PEREIRA, RP.;TOLEDO, RN.; AMARAL, JLG.; GUILHERME, A..Qualificação e quantificação da exposição sonora ambiental em uma unidade de terapia intensiva geral.RevBrasOtorrinolaringol. 2003;69(6):766-71.

POTTER, Patricia; PERRY, Anne Griffin.Fundamentos de Enfermagem. 8 ed. rev. e atual. Rio de Janeiro: Elsecier, 2013. 
ROSA, A. A. C. Avaliação do conforto acústico de consultórios odontológicos. 2003. Dissertação (Mestrado em Engenharia Civil). Faculdade de Engenharia Civil, UNICAMP, 2003.

SANTOS, Nívea Cristina Moreira. Urgência e Emergência para enfermagem: do atendimento pré-hospitalar APH à sala de emergência. 6. ed. rev. e atual. São Paulo: látria, 2010.

SILVA, J.; COSTA, F. S.; SOUZA, R. F.; SOUZA, J. L.; OLIVEIRA, R. S. O ruído causando danos e estresse: possibilidade de atuação para a enfermagem do trabalho. Av. Enferm. 2014; 32(1).

Torres BO, Fernandes MJM, Félix SSS, Costa ICC. A perda auditiva induzida pelo ruído (PAIR) na formação acadêmica: conhecimentos e medidas de prevenção. Odontol Clín Científ. 2007;6(2):151-4.

WEICH, Tainara Milbradt; OURIQUE, Ana Cláudia; TOCHETTO, Tania Maria and FRANCESCHI, Cacineli Marion de.Eficácia de um programa para redução de ruído em unidade de terapia intensiva neonatal. Rev. bras. ter. intensiva [online]. 2011, vol.23, n.3, pp.327-334.

Recebido em: 27/03/2017.

Aprovado em: 11/04/2017. 